\title{
Structure-Composition Relationships in Ternary Solvents Containing Methylbenzoate
}

\author{
S. Aparicio, R. Alcalde, B. García and J. M. Leal* \\ Departamento de Química, Universidad de Burgos, 09001 Burgos, Spain \\ * Corresponding author: jmleal@ubu.es
}

Supporting Information 
TABLE S1: Density, $\rho$, dynamic viscosity, $\eta$, and refractive index, $n_{D}$, of pure compounds at 298.15 $\mathrm{K}$ and $0.1 \mathrm{MPa}$. Measured values in comparison with literature ones

\begin{tabular}{|c|c|c|c|}
\hline & $\rho / \mathrm{g} \mathrm{cm}^{-3}$ & $\eta / \mathrm{mPa} s$ & $n_{D}$ \\
\hline \multirow[t]{3}{*}{ methylbenzoate } & $1.08363^{\mathrm{a}}$ & $1.823^{\mathrm{a}}$ & $1.51466^{\mathrm{a}}$ \\
\hline & $1.08262^{\mathrm{b}}$ & $1.825^{\mathrm{c}}$ & $1.51457^{\mathrm{d}}$ \\
\hline & $1.0836^{\mathrm{c}}$ & & $1.5149^{\mathrm{c}}$ \\
\hline \multirow[t]{2}{*}{ n-hexane } & $0.65498^{\mathrm{a}}$ & $0.330^{\mathrm{a}}$ & $1.37244^{\mathrm{a}}$ \\
\hline & $0.65484^{\mathrm{d}}$ & $0.2949^{\mathrm{e}}$ & $1.37226^{\mathrm{d}}$ \\
\hline \multirow[t]{2}{*}{ 1-chlorohexane } & $0.87356^{\mathrm{a}}$ & $0.704^{\mathrm{a}}$ & $1.41828^{\mathrm{a}}$ \\
\hline & $0.87338^{f}$ & $0.686^{\mathrm{g}}$ & \\
\hline \multirow[t]{2}{*}{ hexanoic acid } & $0.92201^{\mathrm{a}}$ & $2.757^{\mathrm{a}}$ & $1.41510^{\mathrm{a}}$ \\
\hline & $0.9230^{\mathrm{d}}$ & $2.826^{\mathrm{d}}$ & $1.4148^{\mathrm{d}}$ \\
\hline \multirow[t]{2}{*}{ diisopropylether } & $0.71807^{\mathrm{a}}$ & $0.362^{\mathrm{a}}$ & $1.36532^{\mathrm{a}}$ \\
\hline & $0.71854^{\mathrm{d}}$ & $0.379^{\mathrm{d}}$ & $1.3655^{\mathrm{d}}$ \\
\hline
\end{tabular}

${ }^{\mathrm{a}}$ Values obtained in this work; ${ }^{\mathrm{b}}$ Ref. $14 \mathrm{~h} ;{ }^{\mathrm{c}}$ Ref. $14 \mathrm{f} ;{ }^{\mathrm{d}}$ Ref. $22 ;{ }^{\mathrm{e}}$ Ref. $23 ;{ }^{\mathrm{f}}$ Ref. $24 ;{ }^{\mathrm{g}}$ Ref. 25 
TABLE S2: Density, $\rho$, dynamic viscosity, $\eta$, and refractive index, $n_{D}$, for $x$ n-hexane $+(1-x)$ (1-chlorohexane or hexanoic acid or diisopropylether) binary mixtures at 298.15 K and 0.1 MPa

\begin{tabular}{|c|c|c|c|c|c|c|c|c|c|c|c|}
\hline \multicolumn{4}{|c|}{$x$ n-hexane $+(1-x)$ 1-chlorohexane } & \multicolumn{4}{|c|}{$x$ n-hexane $+(1-x)$ hexanoic acid } & \multicolumn{4}{|c|}{$x$ n-hexane $+(1-x)$ diisopropylether } \\
\hline$x$ & $\rho / \mathrm{g} \mathrm{cm}^{-3}$ & $\eta / \mathrm{mPa} \mathrm{s}$ & $n_{D}$ & $x$ & $\rho / \mathrm{g} \mathrm{cm}^{-3}$ & $\eta / \mathrm{mPa} \mathrm{s}$ & $n_{D}$ & $x$ & $\rho / \mathrm{g} \mathrm{cm}^{-3}$ & $\eta / \mathrm{mPa} \mathrm{s}$ & $n_{D}$ \\
\hline 0.0363 & 0.86616 & 0.682 & 1.41673 & 0.0334 & 0.91292 & 2.488 & 1.41399 & 0.0268 & 0.71634 & 0.360 & 1.36543 \\
\hline 0.0978 & 0.85350 & 0.648 & 1.41408 & 0.0851 & 0.89888 & 2.123 & 1.41221 & 0.0978 & 0.71176 & 0.355 & 1.36571 \\
\hline 0.1473 & 0.84322 & 0.622 & 1.41197 & 0.1365 & 0.88493 & 1.828 & 1.41039 & 0.1501 & 0.70840 & 0.351 & 1.36594 \\
\hline 0.2044 & 0.83124 & 0.595 & 1.40951 & 0.1836 & 0.87218 & 1.601 & 1.40868 & 0.2054 & 0.70487 & 0.348 & 1.36618 \\
\hline 0.2477 & 0.82209 & 0.574 & 1.40766 & 0.2301 & 0.85956 & 1.416 & 1.40693 & 0.2522 & 0.70188 & 0.346 & 1.36641 \\
\hline 0.2978 & 0.81144 & 0.552 & 1.40553 & 0.2765 & 0.84701 & 1.258 & 1.40520 & 0.3025 & 0.69867 & 0.345 & 1.36667 \\
\hline 0.3458 & 0.80116 & 0.532 & 1.40350 & 0.3255 & 0.83377 & 1.112 & 1.40326 & 0.3528 & 0.69547 & 0.343 & 1.36696 \\
\hline 0.3975 & 0.79001 & 0.511 & 1.40126 & 0.3732 & 0.82091 & 0.992 & 1.40132 & 0.4035 & 0.69224 & 0.341 & 1.36727 \\
\hline 0.4317 & 0.78259 & 0.499 & 1.39980 & 0.4169 & 0.80914 & 0.897 & 1.39951 & 0.4441 & 0.68965 & 0.340 & 1.36753 \\
\hline 0.4932 & 0.76917 & 0.477 & 1.39710 & 0.4687 & 0.79523 & 0.800 & 1.39732 & 0.4995 & 0.68613 & 0.339 & 1.36791 \\
\hline 0.5403 & 0.75881 & 0.461 & 1.39502 & 0.5159 & 0.78257 & 0.724 & 1.39524 & 0.5485 & 0.68302 & 0.338 & 1.36827 \\
\hline 0.5800 & 0.75001 & 0.448 & 1.39324 & 0.5652 & 0.76939 & 0.656 & 1.39302 & 0.6018 & 0.67965 & 0.337 & 1.36867 \\
\hline 0.6339 & 0.73799 & 0.432 & 1.39079 & 0.6106 & 0.75728 & 0.603 & 1.39091 & 0.6453 & 0.67689 & 0.336 & 1.36901 \\
\hline 0.6854 & 0.72645 & 0.417 & 1.38841 & 0.6657 & 0.74259 & 0.541 & 1.38831 & 0.6925 & 0.67392 & 0.335 & 1.36940 \\
\hline 0.7446 & 0.71306 & 0.400 & 1.38560 & 0.7089 & 0.73107 & 0.503 & 1.38630 & 0.7369 & 0.67113 & 0.335 & 1.36979 \\
\hline 0.7805 & 0.70491 & 0.389 & 1.38385 & 0.7616 & 0.71704 & 0.462 & 1.38371 & 0.7966 & 0.66739 & 0.333 & 1.37033 \\
\hline 0.8256 & 0.69463 & 0.377 & 1.38158 & 0.8098 & 0.70427 & 0.430 & 1.38141 & 0.8252 & 0.66562 & 0.333 & 1.37061 \\
\hline 0.8737 & 0.68365 & 0.364 & 1.37914 & 0.8617 & 0.69057 & 0.398 & 1.37891 & 0.8745 & 0.66257 & 0.332 & 1.37109 \\
\hline 0.9175 & 0.67364 & 0.352 & 1.37688 & 0.9104 & 0.67786 & 0.373 & 1.37656 & 0.9207 & 0.65974 & 0.332 & 1.37158 \\
\hline
\end{tabular}


TABLE S3: Density, $\rho$, dynamic viscosity, $\eta$, and refractive index, $n_{D}$, for the $x_{1}$ n-hexane $+x_{2}$ (1-chlorohexane or hexanoic acid or diisopropylether) $+\left(1-x_{1}-x_{2}\right)$ methylbenzoate (MB) ternary mixtures at 298.15 $\mathrm{K}$ and $0.1 \mathrm{MPa}$

\begin{tabular}{|c|c|c|c|c|c|c|c|c|c|c|c|c|c|c|}
\hline \multicolumn{5}{|c|}{$x_{1} \mathrm{n}$-hexane $+x_{2} 1$-chlorohexane $+\left(1-x_{1}-x_{2}\right) \mathrm{MB}$} & \multicolumn{5}{|c|}{$x_{1}$ n-hexane $+x_{2}$ hexanoic acid $+\left(1-x_{1}-x_{2}\right)$ MB } & \multicolumn{5}{|c|}{$x_{1} \mathrm{n}$-hexane $+x_{2}$ diisopropylether $+\left(1-x_{1}-x_{2}\right) \mathrm{MB}$} \\
\hline$x_{1}$ & $x_{2}$ & $\rho / \mathrm{g} \mathrm{cm}^{-3}$ & $\eta / \mathrm{mPa} \mathrm{s}$ & $n_{D}$ & $x_{1}$ & $x_{2}$ & $\rho / \mathrm{g} \mathrm{cm}^{-3}$ & $\eta / \mathrm{mPa} \mathrm{s}$ & $n_{D}$ & $x_{1}$ & $x_{2}$ & $\rho / \mathrm{g} \mathrm{cm}^{-3}$ & $\eta / \mathrm{mPa} \mathrm{s}$ & $n_{D}$ \\
\hline 0.0918 & 0.8088 & 0.87372 & & 1.42307 & 0.0947 & 0.8143 & 0.91074 & 1.988 & 1.42132 & 0.0926 & 0.8139 & 0.74617 & 0.405 & \\
\hline 0.0817 & 0.7252 & 0.89442 & 0.744 & 1.43229 & 0.0831 & 0.7207 & 0.93149 & 1.966 & 1.43216 & 0.0821 & 0.7287 & 0.78177 & & \\
\hline 0.0721 & 0.6324 & 0.91705 & 0.803 & 1.44258 & 0.0717 & 0.6299 & 0.95139 & 1.954 & 1.44294 & 0.0728 & 0.6318 & 0.82136 & 0.525 & \\
\hline 0.0622 & 0.5377 & 0.94067 & 0.881 & 1.45365 & 0.0624 & 0.5375 & 0.97058 & 1.935 & 1.45409 & 0.0614 & 0.5434 & 0.85890 & 0.612 & \\
\hline 0.0510 & 0.4465 & 0.96456 & 0.978 & 1.46347 & 0.0515 & 0.4462 & 0.99024 & 1.927 & 1.46489 & 0.0529 & 0.4457 & 0.89857 & 0.732 & \\
\hline 0.0335 & 0.3802 & 0.98604 & 1.082 & 1.47238 & 0.0416 & 0.3550 & 1.00935 & 1.915 & 1.47489 & 0.0427 & 0.3569 & 0.93542 & 0.879 & 1.46181 \\
\hline 0.0305 & 0.2657 & 1.01276 & 1.236 & 1.48404 & 0.0306 & 0.2633 & 1.02897 & 1.907 & 1.48562 & 0.0319 & 0.2689 & 0.97231 & 1.058 & 1.47816 \\
\hline 0.0196 & 0.1763 & 1.03611 & & 1.49446 & 0.0208 & 0.1722 & 1.04778 & & 1.49605 & 0.0207 & 0.1736 & 1.01193 & & 1.49182 \\
\hline 0.0914 & 0.8064 & 0.87439 & & 1.42355 & 0.0099 & 0.0904 & 1.06545 & 1.862 & 1.50504 & 0.0101 & 0.0810 & 1.05006 & 1.567 & 1.50346 \\
\hline 0.0930 & 0.8099 & 0.87348 & 0.687 & 1.42293 & 0.1815 & 0.7229 & 0.88837 & 1.545 & 1.41855 & 0.0867 & 0.7203 & & 0.460 & \\
\hline 0.1523 & 0.7456 & 0.86166 & 0.656 & 1.42157 & 0.1661 & 0.6373 & 0.90909 & 1.549 & 1.42994 & 0.1281 & 0.7637 & 0.74909 & 0.412 & 1.38373 \\
\hline 0.1582 & 0.6431 & 0.87953 & 0.693 & 1.43015 & 0.1491 & 0.5542 & 0.93052 & 1.568 & 1.44152 & 0.1565 & 0.6518 & 0.77769 & 0.454 & 1.39760 \\
\hline 0.1359 & 0.5678 & 0.90403 & 0.756 & 1.44062 & 0.1267 & 0.4751 & 0.95301 & 1.602 & 1.45171 & 0.1356 & 0.5667 & 0.81804 & 0.526 & 1.41712 \\
\hline 0.1197 & 0.4812 & 0.92877 & 0.834 & 1.45157 & 0.1065 & 0.3945 & 0.97497 & 1.640 & 1.46373 & 0.1222 & 0.4799 & 0.85569 & & 1.43267 \\
\hline 0.0983 & 0.3982 & 0.95511 & 0.936 & 1.46194 & 0.0833 & 0.3129 & 0.99825 & & 1.47441 & 0.0993 & 0.3991 & 0.89583 & 0.733 & 1.44534 \\
\hline 0.0834 & 0.3138 & 0.97937 & 1.047 & 1.47301 & 0.0621 & 0.2323 & 1.02049 & & 1.48524 & 0.0879 & 0.2883 & 0.94095 & 0.907 & 1.46853 \\
\hline 0.0574 & 0.2291 & & 1.225 & 1.48566 & 0.0414 & 0.1502 & 1.04254 & & 1.49572 & 0.0650 & 0.2294 & 0.97262 & 1.051 & 1.47813 \\
\hline 0.0196 & 0.0820 & 1.05694 & 1.580 & 1.50420 & 0.2705 & 0.6335 & 0.86476 & 1.216 & 1.41536 & 0.1706 & 0.7273 & & 0.407 & 1.38292 \\
\hline 0.2888 & 0.6113 & 0.83282 & 0.585 & 1.41667 & 0.2446 & 0.5554 & 0.88881 & 1.258 & & 0.1237 & 0.4665 & 0.85972 & 0.623 & 1.43164 \\
\hline 0.2366 & 0.5645 & 0.86315 & 0.647 & 1.42756 & 0.2122 & 0.4898 & 0.91340 & 1.320 & 1.43889 & 0.2718 & 0.6256 & & 0.399 & 1.38352 \\
\hline 0.2137 & 0.4912 & 0.88791 & 0.706 & 1.43805 & 0.1796 & 0.4180 & 0.93941 & 1.426 & 1.45246 & 0.2437 & 0.5647 & 0.77206 & 0.445 & 1.39824 \\
\hline 0.1852 & 0.4212 & 0.91385 & 0.782 & 1.44943 & 0.1462 & 0.3474 & 0.96556 & 1.494 & 1.46339 & 0.2113 & 0.4927 & 0.81269 & 0.512 & 1.41443 \\
\hline 0.1498 & 0.3468 & 0.94439 & 0.889 & 1.46162 & 0.1230 & 0.2734 & 0.98761 & 1.543 & 1.47410 & 0.1803 & 0.4080 & 0.85741 & & \\
\hline 0.1165 & 0.2789 & 0.97295 & 1.015 & 1.47288 & 0.0842 & 0.2035 & 1.01565 & 1.641 & 1.48537 & 0.1498 & 0.3467 & 0.89264 & 0.712 & 1.44632 \\
\hline 0.0912 & 0.2059 & 0.99978 & 1.160 & 1.48376 & 0.1834 & 0.5078 & 0.92325 & 1.427 & 1.44120 & 0.1140 & 0.2700 & 0.93627 & & 1.46687 \\
\hline
\end{tabular}


TABLE S3: Continued

\begin{tabular}{|c|c|c|c|c|c|c|c|c|c|c|c|c|c|c|}
\hline$x_{1}$ & $x_{2}$ & $\rho / \mathrm{g} \mathrm{cm}^{-3}$ & $\eta / \mathrm{mPa} \mathrm{s}$ & $n_{D}$ & $x_{1}$ & $x_{2}$ & $\rho / \mathrm{g} \mathrm{cm}^{-3}$ & $\eta / \mathrm{mPa} \mathrm{s}$ & $n_{D}$ & $x_{1}$ & $x_{2}$ & $\rho / \mathrm{g} \mathrm{cm}^{-3}$ & $\eta / \mathrm{mPa} \mathrm{s}$ & $n_{D}$ \\
\hline 0.0602 & 0.1267 & 1.02997 & 1.362 & 1.49581 & 0.3638 & 0.5382 & 0.83934 & & 1.41214 & 0.0959 & 0.2017 & 0.96914 & 1.029 & 1.47719 \\
\hline 0.3581 & 0.5403 & 0.81795 & 0.560 & 1.41280 & 0.3240 & 0.4751 & 0.86716 & & 1.42429 & 0.0635 & 0.1362 & 1.00716 & 1.246 & 1.49223 \\
\hline 0.3213 & 0.4822 & 0.84462 & 0.611 & 1.42386 & 0.2806 & 0.4191 & 0.89517 & 1.146 & 1.43721 & 0.0321 & 0.0647 & 1.04664 & & 1.50305 \\
\hline 0.2863 & 0.4200 & 0.87180 & 0.671 & 1.43468 & 0.2414 & 0.3565 & 0.92258 & 1.212 & & 0.1489 & 0.4353 & 0.86033 & & \\
\hline 0.2452 & 0.3654 & 0.90042 & 0.754 & 1.44575 & 0.2025 & 0.2954 & 0.94962 & 1.296 & 1.46114 & 0.3631 & 0.5338 & 0.73194 & 0.400 & 1.38394 \\
\hline 0.1983 & 0.2987 & 0.93444 & 0.859 & 1.45957 & 0.1582 & 0.2334 & 0.97892 & 1.416 & 1.47437 & 0.3199 & 0.4808 & 0.76978 & 0.450 & 1.39939 \\
\hline 0.1641 & 0.2354 & 0.96217 & 0.978 & 1.47204 & 0.1186 & 0.1725 & 1.00590 & 1.520 & 1.48504 & 0.2881 & 0.4069 & 0.81092 & 0.518 & 1.41834 \\
\hline 0.1170 & 0.1716 & 0.99604 & 1.151 & 1.48362 & 0.0832 & 0.1090 & 1.03131 & 1.606 & 1.49514 & 0.2412 & 0.3593 & 0.84881 & 0.598 & 1.43201 \\
\hline 0.0410 & 0.0592 & 1.05309 & 1.559 & 1.50482 & 0.0393 & 0.0874 & 1.05372 & 1.756 & 1.50267 & 0.2008 & 0.2923 & 0.89141 & 0.713 & 1.44893 \\
\hline 0.4404 & 0.4577 & 0.80093 & 0.523 & 1.40902 & 0.4579 & 0.4452 & 0.81459 & 0.801 & 1.40781 & 0.1610 & 0.2322 & 0.92987 & 0.854 & 1.46332 \\
\hline 0.4070 & 0.3972 & 0.82601 & 0.565 & 1.42045 & 0.4055 & 0.3911 & 0.84604 & 0.878 & 1.42188 & 0.1185 & 0.1763 & 0.96887 & 1.030 & 1.47845 \\
\hline 0.3525 & 0.3475 & 0.85890 & 0.633 & 1.43247 & 0.3500 & 0.3476 & 0.87689 & 0.952 & 1.43451 & 0.0296 & 0.0786 & 1.04268 & & 1.50187 \\
\hline 0.3039 & 0.2987 & 0.89029 & 0.711 & 1.44488 & 0.3015 & 0.2970 & 0.90639 & 1.037 & 1.44728 & 0.1826 & 0.4196 & & 0.611 & 1.42938 \\
\hline 0.2495 & 0.2485 & 0.92341 & 0.816 & 1.45958 & 0.2521 & 0.2424 & & 1.142 & 1.46027 & 0.4570 & 0.4410 & 0.72579 & 0.394 & 1.38404 \\
\hline 0.2010 & 0.1913 & & 0.944 & 1.47166 & 0.2020 & 0.1949 & & 1.257 & 1.47344 & 0.4084 & 0.3904 & 0.76494 & 0.441 & 1.40003 \\
\hline 0.1487 & 0.1419 & 0.98898 & & 1.48444 & 0.1523 & 0.1398 & 0.99662 & 1.368 & 1.48402 & 0.3568 & 0.3447 & & 0.504 & 1.41887 \\
\hline 0.0999 & 0.0869 & 1.02187 & & & 0.0999 & 0.0917 & 1.02691 & 1.520 & 1.49614 & 0.3011 & 0.2882 & 0.84898 & 0.597 & 1.43668 \\
\hline 0.5490 & 0.3526 & 0.77577 & 0.486 & 1.40418 & 0.5483 & 0.3551 & & 0.677 & 1.40526 & 0.2547 & 0.2437 & 0.88585 & 0.697 & 1.44818 \\
\hline 0.4845 & 0.3210 & 0.80886 & 0.537 & 1.41646 & 0.4859 & 0.3121 & & 0.746 & 1.41945 & 0.1898 & 0.1958 & & 0.862 & 1.46825 \\
\hline 0.4234 & 0.2746 & 0.84408 & 0.614 & 1.43032 & 0.4175 & 0.2787 & 0.85898 & 0.823 & 1.43201 & 0.1490 & 0.1479 & 0.96623 & 1.006 & 1.47993 \\
\hline 0.3651 & 0.2333 & 0.87724 & 0.687 & 1.44382 & 0.3605 & 0.2349 & 0.89087 & 0.908 & 1.44513 & 0.0968 & 0.0895 & 1.01016 & 1.254 & 1.49291 \\
\hline 0.3008 & 0.1937 & 0.91314 & 0.800 & & 0.3000 & 0.1947 & 0.92367 & 1.012 & & 0.5493 & 0.3543 & 0.71764 & 0.390 & 1.38416 \\
\hline 0.2380 & 0.1541 & 0.94844 & 0.930 & & 0.2425 & 0.1505 & 0.95599 & 1.125 & 1.47154 & 0.4776 & 0.3206 & 0.76056 & 0.441 & 1.40349 \\
\hline 0.1788 & 0.1104 & 0.98317 & 1.091 & 1.48546 & 0.1838 & 0.1098 & 0.98820 & 1.267 & 1.48483 & 0.4245 & 0.2735 & 0.80112 & 0.503 & 1.42090 \\
\hline 0.0619 & 0.0396 & 1.04864 & 1.525 & 1.50481 & 0.6390 & 0.2627 & & 0.559 & 1.40046 & 0.3674 & 0.2335 & 0.84017 & 0.585 & 1.43354 \\
\hline 0.0961 & 0.0861 & 1.02436 & 1.336 & 1.49684 & 0.5685 & 0.2292 & 0.80195 & 0.618 & 1.41482 & 0.3019 & 0.1935 & 0.88405 & 0.698 & 1.44692 \\
\hline 0.6343 & 0.2646 & 0.75763 & 0.456 & 1.40051 & 0.4914 & 0.2055 & & 0.702 & & 0.2374 & 0.1555 & 0.92553 & 0.840 & 1.46346 \\
\hline
\end{tabular}




\section{TABLE S3: Continued}

\begin{tabular}{|c|c|c|c|c|c|c|c|c|c|c|c|c|c|c|}
\hline \multicolumn{5}{|c|}{$x_{1}$ n-hexane $+x_{2}$ 1-chlorohexane $+\left(1-x_{1}-x_{2}\right) \mathrm{MB}$} & \multicolumn{5}{|c|}{$x_{1}$ n-hexane $+x_{2}$ hexanoic acid $+\left(1-x_{1}-x_{2}\right) \mathrm{MB}$} & \multicolumn{5}{|c|}{$x_{1} \mathrm{n}$-hexane $+x_{2}$ diisopropylether $+\left(1-x_{1}-x_{2}\right) \mathrm{MB}$} \\
\hline$x_{1}$ & $x_{2}$ & $\rho / \mathrm{g} \mathrm{cm}^{-3}$ & $\eta / \mathrm{mPa} \mathrm{s}$ & $n_{D}$ & $x_{1}$ & $x_{2}$ & $\rho / \mathrm{g} \mathrm{cm}^{-3}$ & $\eta / \mathrm{mPa} \mathrm{s}$ & $n_{D}$ & $x_{1}$ & $x_{2}$ & $\rho / \mathrm{g} \mathrm{cm}^{-3}$ & $\eta / \mathrm{mPa} \mathrm{s}$ & $n_{D}$ \\
\hline 0.5702 & 0.2321 & 0.79047 & 0.500 & 1.41299 & 0.4246 & 0.1765 & 0.87275 & 0.783 & 1.44460 & 0.1791 & 0.1091 & 0.96757 & 1.022 & 1.48001 \\
\hline 0.4964 & 0.2019 & 0.82824 & & 1.42771 & 0.3516 & 0.1441 & 0.91003 & 0.899 & 1.45683 & 0.6358 & 0.2644 & 0.71364 & 0.388 & 1.38500 \\
\hline 0.4246 & 0.1726 & 0.86452 & 0.648 & 1.44055 & 0.2846 & 0.1118 & 0.94422 & & 1.46989 & 0.5676 & 0.2364 & 0.75256 & 0.432 & 1.40148 \\
\hline 0.3574 & 0.1427 & & 0.758 & & 0.2124 & 0.0914 & 0.97887 & 1.178 & 1.48176 & 0.4957 & 0.2026 & 0.79627 & 0.495 & 1.41825 \\
\hline 0.2831 & 0.1116 & 0.93898 & 0.882 & 1.46944 & 0.1400 & 0.0409 & 1.01788 & 1.359 & 1.49573 & 0.4258 & 0.1785 & & 0.572 & 1.43441 \\
\hline 0.2132 & 0.0858 & 0.97380 & 1.035 & & 0.0395 & 0.0303 & 1.06237 & 1.697 & 1.50761 & 0.3565 & 0.1400 & & & 1.45080 \\
\hline 0.1387 & 0.0830 & 1.00624 & 1.221 & 1.49130 & 0.3051 & 0.1092 & 0.93572 & 0.972 & 1.46736 & 0.2875 & 0.0816 & 0.93099 & 0.859 & 1.46936 \\
\hline 0.7244 & 0.1762 & 0.73661 & 0.426 & 1.39605 & 0.7294 & 0.1752 & 0.74139 & 0.485 & 1.39595 & 0.2099 & 0.0805 & 0.96505 & 1.004 & 1.48048 \\
\hline 0.6499 & 0.1546 & 0.77215 & 0.475 & 1.40918 & 0.6459 & 0.1515 & 0.78135 & 0.542 & 1.41225 & 0.1381 & 0.0611 & 1.00271 & & 1.49310 \\
\hline 0.5685 & 0.1325 & 0.81153 & 0.540 & 1.42532 & 0.5680 & 0.1339 & 0.81787 & 0.604 & 1.42648 & 0.0661 & 0.0352 & 1.04226 & & 1.50292 \\
\hline 0.4855 & 0.1117 & 0.85154 & 0.625 & 1.44018 & 0.4888 & 0.1119 & 0.85562 & 0.687 & 1.44071 & 0.1402 & 0.0619 & 1.00152 & 1.195 & 1.49126 \\
\hline 0.4086 & 0.0932 & 0.88830 & 0.720 & 1.45153 & 0.4053 & 0.0933 & 0.89507 & 0.800 & 1.45647 & 0.7374 & 0.1628 & 0.70675 & 0.384 & 1.38593 \\
\hline 0.3187 & 0.0804 & 0.92973 & & & 0.3204 & 0.0799 & 0.93377 & 0.943 & 1.46860 & 0.6545 & 0.1486 & 0.74729 & 0.428 & 1.40280 \\
\hline 0.2401 & 0.0599 & 0.96808 & & 1.48034 & 0.2422 & 0.0584 & 0.97130 & 1.102 & 1.48139 & 0.5698 & 0.1274 & 0.79117 & 0.490 & 1.42071 \\
\hline 0.4974 & 0.2022 & 0.82774 & 0.575 & 1.42704 & 0.1584 & 0.0408 & 1.01000 & 1.318 & 1.49352 & 0.4883 & 0.1068 & 0.83405 & 0.573 & 1.44008 \\
\hline 0.8140 & 0.1253 & & 0.402 & & 0.8138 & 0.0893 & 0.71903 & 0.421 & 1.39087 & 0.4032 & 0.0893 & & 0.682 & 1.45312 \\
\hline 0.7237 & 0.0822 & 0.75599 & 0.447 & 1.40650 & 0.7274 & 0.0800 & 0.75787 & & 1.40750 & 0.3180 & 0.0827 & 0.91726 & 0.809 & 1.46454 \\
\hline 0.6542 & 0.0672 & 0.78828 & 0.491 & 1.41811 & 0.6398 & 0.0692 & & & 1.42225 & 0.2177 & 0.0555 & 0.95985 & 0.977 & 1.47948 \\
\hline 0.5323 & 0.0618 & 0.84158 & 0.601 & 1.43697 & 0.5470 & 0.0603 & 0.83913 & 0.611 & & 0.8115 & 0.0907 & 0.70161 & 0.379 & 1.38606 \\
\hline 0.4408 & 0.0518 & 0.88357 & 0.706 & 1.45554 & 0.4328 & 0.0515 & 0.88981 & 0.746 & 1.45633 & 0.7236 & 0.0824 & 0.74165 & 0.422 & \\
\hline 0.3603 & 0.0404 & 0.92046 & 0.829 & 1.46766 & 0.3621 & 0.0400 & & 0.857 & & 0.6281 & 0.0706 & 0.78750 & 0.484 & 1.42233 \\
\hline 0.2580 & 0.0304 & 0.96686 & 1.020 & 1.48409 & 0.2691 & 0.0306 & 0.96413 & 1.026 & 1.48058 & 0.5405 & 0.0609 & 0.82908 & 0.559 & 1.43629 \\
\hline 0.1812 & 0.0205 & 1.00202 & 1.217 & 1.49374 & 0.1550 & 0.0213 & & 1.312 & 1.49641 & 0.4461 & 0.0516 & & 0.663 & 1.45126 \\
\hline \multirow[t]{4}{*}{0.0450} & 0.0108 & & & 1.50960 & 0.0668 & 0.0203 & 1.05238 & 1.594 & 1.50565 & 0.3582 & 0.0402 & 0.91528 & 0.791 & 1.46666 \\
\hline & & & & & & & & & & 0.2729 & 0.0301 & 0.95625 & 0.951 & 1.47961 \\
\hline & & & & & & & & & & 0.1761 & 0.0199 & 1.00140 & & 1.49262 \\
\hline & & & & & & & & & & 0.0916 & 0.0098 & 1.04167 & 1.458 & 1.50342 \\
\hline
\end{tabular}


TABLE S4: $A_{i}$ coefficients of eq 1 and standard deviation, $\sigma$, for $x$ n-hexane $+(1-x)$ (1-chlorohexane or hexanoic acid or diisopropylether) binary mixtures at 298.15 $\mathrm{K}$ and $0.1 \mathrm{MPa}$. Excess molar volume, $V_{m}^{E}\left(\mathrm{~cm}^{3} \mathrm{~mol}^{-1}\right)$, mixing viscosity, $\Delta_{m i x} \eta$ (mPa s), and mixing refractive index, $\Delta_{m i x} n_{D}$

\begin{tabular}{|c|c|c|c|c|c|c|}
\hline \multirow[b]{2}{*}{ Property } & \multicolumn{6}{|c|}{$x$ n-hexane $+(1-x)$ 1-chlorohexane } \\
\hline & $A_{0}$ & $A_{1}$ & $A_{2}$ & $A_{3}$ & $A_{4}$ & $\sigma$ \\
\hline$V_{m}^{E}$ & -0.5468 & 0.2932 & 0.5098 & 0.6174 & & 0.0018 \\
\hline$\Delta_{m i x} \eta$ & -0.169 & 0.064 & & & & 0.000 \\
\hline \multirow[t]{2}{*}{$\Delta_{m i x} n_{D}$} & 0.00579 & 0.00380 & 0.00011 & -0.00088 & & 0.00001 \\
\hline & \multicolumn{6}{|c|}{$x$ n-hexane $+(1-x)$ hexanoic acid } \\
\hline Property & $A_{0}$ & $A_{1}$ & $A_{2}$ & $A_{3}$ & $A_{4}$ & $\sigma$ \\
\hline$V_{m}^{E}$ & -0.8152 & 0.1413 & 0.2484 & 0.8957 & 0.7179 & 0.0012 \\
\hline$\Delta_{m i x} \eta$ & -3.175 & 1.702 & -0.894 & 0.458 & & 0.002 \\
\hline \multirow[t]{2}{*}{$\Delta_{m i x} n_{D}$} & 0.00866 & -0.00283 & -0.00308 & -0.00092 & & 0.00003 \\
\hline & \multicolumn{6}{|c|}{$x$ n-hexane $+(1-x)$ diisopropylether } \\
\hline Property & $A_{0}$ & $A_{1}$ & $A_{2}$ & $A_{3}$ & $A_{4}$ & $\sigma$ \\
\hline$V_{m}^{E}$ & 1.3230 & 0.2310 & 0.2028 & & & 0.0009 \\
\hline$\Delta_{m i x} \eta$ & -0.028 & 0.019 & -0.0007 & & & 0.000 \\
\hline$\Delta_{m i x} n_{D}$ & 0.06227 & 0.09695 & 0.05924 & -0.01659 & -0.03620 & 0.00008 \\
\hline
\end{tabular}


TABLE S5: $B_{i}$ coefficients of eq 2 and standard deviation, $\sigma$, for the $x_{1}$ n-hexane $+x_{2}$ (1chlorohexane or hexanoic acid or diisopropylether) $+\left(1-x_{1}-x_{2}\right)$ methylbenzoate (MB) ternary systems at 298.15 $\mathrm{K}$ and 0.1 MPa. Excess molar volume, $V_{m, T E R}^{E}\left(\mathrm{~cm}^{3} \mathrm{~mol}^{-1}\right)$, mixing viscosity, $\Delta_{m i x} \eta_{T E R}(\mathrm{mPa}$ s), and mixing refractive index, $\Delta_{m i x} n_{D, T E R}$

\begin{tabular}{|c|c|c|c|c|}
\hline Property & $B_{0}$ & $B_{1}$ & $B_{2}$ & $\sigma$ \\
\hline & \multicolumn{4}{|c|}{$x_{1}$ n-hexane $+x_{2} 1$-chlorohexane $+\left(1-x_{1}-x_{2}\right) \mathrm{MB}$} \\
\hline$V_{m, T E R}^{E}$ & -3.1264 & 1.2466 & 7.0834 & 0.0349 \\
\hline$\Delta_{m i x} \eta_{T E R}$ & 0.523 & 0.929 & -0.723 & 0.007 \\
\hline \multirow[t]{2}{*}{$\Delta_{m i x} n_{D, T E R}$} & 0.0382 & -0.1451 & -0.0500 & 0.0008 \\
\hline & \multicolumn{4}{|c|}{$x_{1}$ n-hexane $+x_{2}$ hexanoic acid $+\left(1-x_{1}-x_{2}\right) \mathrm{MB}$} \\
\hline$V_{m, T E R}^{E}$ & -1.0838 & -7.5454 & 4.5791 & 0.0306 \\
\hline$\Delta_{m i x} \eta_{T E R}$ & -4.467 & 5.167 & 0.893 & 0.009 \\
\hline \multirow[t]{2}{*}{$\Delta_{m i x} n_{D, T E R}$} & 0.0596 & -0.0334 & -0.0899 & 0.0006 \\
\hline & \multicolumn{4}{|c|}{$x_{1}$ n-hexane $+x_{2}$ diisopropylether $+\left(1-x_{1}-x_{2}\right) \mathrm{MB}$} \\
\hline$V_{m, T E R}^{E}$ & -5.6673 & 3.9890 & 5.4597 & 0.0373 \\
\hline$\Delta_{m i x} \eta_{T E R}$ & -0.108 & 1.639 & 1.750 & 0.005 \\
\hline$\Delta_{m i x} n_{D, T E R}$ & 0.1812 & 0.0180 & -1.0241 & 0.0016 \\
\hline
\end{tabular}


TABLE S6: Coordinates for the maxima and minima of the ternary contributions to excess molar volume, $\left(V_{m, T E R}^{E}-V_{m, B I N}^{E}\right)\left(\mathbf{c m}^{\mathbf{3}} \mathbf{m o l}^{-\mathbf{1}}\right)$, mixing viscosity, $\left(\Delta_{m i x} \eta_{T E R}-\Delta_{m i x} \eta_{B I N}\right)(\mathbf{m P a} \mathbf{s})$ and mixing refractive index, $\left(\Delta_{m i x} n_{D, T E R}-\Delta_{m i x} n_{D, B I N}\right)$ for the $x_{1} \mathbf{n}$-hexane $+\boldsymbol{x}_{2}$ (1-chlorohexane or hexanoic acid or diisopropylether $)+(1-$ $x_{1}-x_{2}$ ) methylbenzoate (MB) ternary systems at 298.15 K and 0.1 MPa

\begin{tabular}{|c|c|c|c|c|c|c|}
\hline & \multicolumn{2}{|c|}{$\left(V_{m, T E R}^{E}-V_{m, B I N}^{E}\right)$} & \multicolumn{2}{|c|}{$\left(\Delta_{m i x} \eta_{T E R}-\Delta_{m i x} \eta_{B I N}\right)$} & \multicolumn{2}{|c|}{$\left(\Delta_{m i x} n_{D, T E R}-\Delta_{m i x} n_{D, B I N}\right)$} \\
\hline & \multicolumn{6}{|c|}{$x_{1}$ n-hexane $+x_{2} 1$-chlorohexane $+\left(1-x_{1}-x_{2}\right) \mathrm{MB}$} \\
\hline & Position & Amount & Position & Amount & Position & Amount \\
\hline Maximum & $\begin{array}{l}x_{1}=0.1940 \\
x_{2}=0.6371\end{array}$ & 0.0340 & $\begin{array}{l}x_{1}=0.4650 \\
x_{2}=0.2350\end{array}$ & 0.026 & $\begin{array}{l}x_{1}=0.0892 \\
x_{2}=0.2085\end{array}$ & 0.00019 \\
\hline Minimum & $\begin{array}{l}x_{1}=0.3547 \\
x_{2}=0.1542\end{array}$ & -0.0428 & $\begin{array}{l}x_{1}=0.0405 \\
x_{2}=0.8722\end{array}$ & -0.002 & $\begin{array}{l}x_{1}=0.5241 \\
x_{2}=0.2653\end{array}$ & -0.00150 \\
\hline
\end{tabular}

$x_{1}$ n-hexane $+x_{2}$ hexanoic acid $+\left(1-x_{1}-x_{2}\right) \mathrm{MB}$

\begin{tabular}{|c|c|c|c|c|c|c|}
\hline & Position & Amount & Position & Amount & Position & Amount \\
\hline Maximum & $\begin{array}{l}x_{1}=0.0969 \\
x_{2}=0.6945\end{array}$ & 0.0192 & $\begin{array}{l}x_{1}=0.9022 \\
x_{2}=0.0533\end{array}$ & 0.005 & $\begin{array}{l}x_{1}=0.3171 \\
x_{2}=0.2009\end{array}$ & 0.00095 \\
\hline Minimum & $\begin{array}{l}x_{1}=0.5262 \\
x_{2}=0.2062\end{array}$ & -0.1193 & $\begin{array}{l}x_{1}=0.2359 \\
x_{2}=0.3596\end{array}$ & -0.100 & $\begin{array}{l}x_{1}=0.1593 \\
x_{2}=0.7318\end{array}$ & -0.00015 \\
\hline
\end{tabular}

$x_{1} \mathrm{n}$-hexane $+x_{2}$ diisopropylether $+\left(1-x_{1}-x_{2}\right) \mathrm{MB}$

\begin{tabular}{|c|c|c|c|c|c|c|}
\hline & Position & Amount & Position & Amount & Position & Amount \\
\hline Maximum & & & & & $\begin{array}{l}x_{1}=0.4765 \\
x_{2}=0.0830\end{array}$ & 0.00183 \\
\hline Minimum & $\begin{array}{l}x_{1}=0.2887 \\
x_{2}=0.2545\end{array}$ & -0.1049 & $\begin{array}{l}x_{1}=0.3711 \\
x_{2}=0.3846\end{array}$ & 0.041 & $\begin{array}{l}x_{1}=0.2213 \\
x_{2}=0.5552\end{array}$ & -0.01053 \\
\hline
\end{tabular}

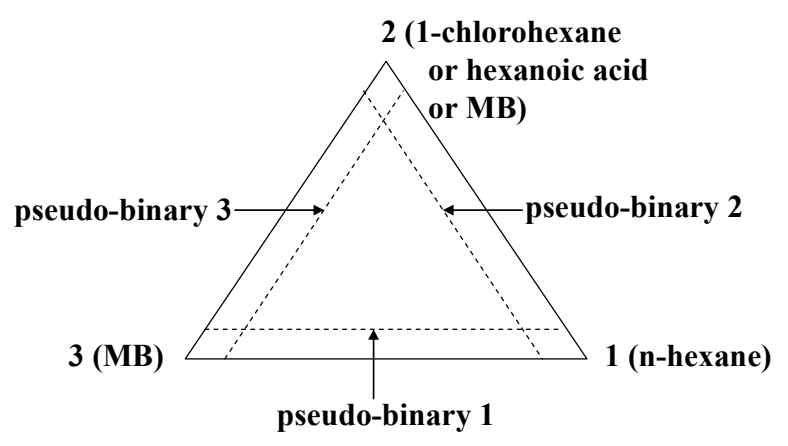

Figure I. Scheme of pseudo-binary mixtures for KB analysis. 